\title{
Photonic Crystal Frequency Demultiplexer Design for Electromagnetic Wave using FDTD and MEMD
}

\author{
Ran Dong ${ }^{1, *}$, Daisuke Shigeta ${ }^{1}$, Yoshihisa Fujita ${ }^{2}$, Soichiro Ikuno $^{1}$ \\ ${ }^{1}$ School of Computer Science, Tokyo University of Technology \\ ${ }^{2}$ College of Information Science and Engineering, Ritsumeikan University \\ *randong@stf.teu.ac.jp
}

Received: October 1, 2021; Accepted: January 24, 2022; Published: February 8, 2022

\begin{abstract}
Photonic crystals are widely employed in industry fields due to their bandgap property, which can confine and propagate electromagnetic waves inside the structure as a photonic waveguide. As this property can be adopted to propagate distinct frequency waves by changing the photonic structure, photonic crystals are also applied in frequency demultiplexer design. The finite-difference time-domain (FDTD) method is commonly applied to simulate electromagnetic wave propagations in photonic crystals, helping determine the desired bandgaps for frequency demultiplexers. Meanwhile, the multivariate empirical mode decomposition (MEMD) nonlinearly decomposes multivariate signals in the instantaneous frequency domain. Therefore, MEMD can verify and visualize the designed frequency demultiplexer made of photonic crystals by considering simulation results as a multi-channel signal. This research aims to propose a method to design and evaluate frequency demultiplexers using FDTD and MEMD. In this paper, photonic crystal bandgaps are adopted to design a frequency demultiplexer to separate two different frequency electromagnetic waves. Then, MEMD is employed to the result of frequency demultiplexer propagation simulated by FDTD. Our results reveal that the frequency demultiplexer made of photonic crystals can be designed using the bandgap properties, and its simulation results by FDTD method can be verified and visualized in the instantaneous frequency domain using MEMD.
\end{abstract}

Keywords: Finite-difference time-domain, Photonic crystal, Frequency demultiplexer, Empirical mode decomposition, Hilbert transform

\section{Introduction}

Photonic crystals are artificial structures consisting of two or more materials with different permittivities, arranged in a $2 \mathrm{D}$ or $3 \mathrm{D}$ periodic pattern with a size smaller than the wavelength [1]. When crystals are set at equal intervals for causing permittivities to change periodically, a region is obtained that electromagnetic waves cannot be allowed to exist in the region for a specific frequency. This property is called the photonic bandgap [1]. Thus, 
using this bandgap property by defecting rows of the crystal structure, the electromagnetic wave only can propagate in the defective area. The photonic bandgap is the essential feature of the photonic crystal, and it can be employed to design photonic crystal waveguides. Furthermore, because the bandgap depends on the frequency of the input wave and the photonic crystal structure, the bandgap property can also be applied to design frequency demultiplexers made of photonic crystals. For example, Rostami et al. [2] proposed a new method to design demultiplexers using photonic crystals. Although the frequency demultiplexer made of photonic crystals could separate the distinct wave, the nonlinear reflections and penetrations occur in the demultiplexer, making it difficult to evaluate the performance of the frequency demultiplexer. However, limited research has focused on the analysis of the demultiplexer performance in the frequency domain.

Electromagnetic wave propagation in photonic crystals can be simulated by the finitedifference time-domain (FDTD) method. FDTD is widely employed in electromagnetic wave simulations to obtain the spatial distribution of electromagnetic fields by solving the Maxwell equations in time series [3]. There are plenty of researches that have been conducted in designing devices using FDTD method. Kawaguchi et al. [4] adopted FDTD method in real-time simulation to design microwave devices. Itoh et al. [5] proposed a method to improve the simulation efficiency of FDTD method in simulating electromagnetic wave propagation. There are also many types of research to simulate photonic crystal propagation using FDTD based on the bandgap property, such as [6]. This research proposed a method to simulate the propagation of electromagnetic waves in $3 \mathrm{D}$ photonic crystals using FDTD method. Moreover, various studies have additionally conducted frequency analysis of the wave propagation simulated by FDTD. Fujita et al. [7] employed FDTD method for electromagnetic wave propagation in a corrugated waveguide and showed that reflection waves occur in different frequency modes. Moreover, some researches have been performed to analyze wave propagation using the Fourier transform (FT) in the frequency domain, such as $[8,9,10]$. However, FT can only deal with linear modes regarding the unsatisfactory performance in wave propagation analysis.

The empirical mode decomposition (EMD) proposed by Huang et al. [11] has recently demonstrated excellent performance in mode decomposition of nonlinear signals. After EMD had been proposed, this method was expanded from one variate to bivariate, and trivariate $[12,13]$. Furthermore, the multivariate empirical mode decomposition (MEMD) has also been proposed and is used for analyzing signals with multi-channels [14]. Therefore, this study aims to design a frequency demultiplexer made of photonic crystals using the bandgap property and propose a method to analyze and evaluate the FDTD simulation result of the demultiplexer using MEMD.

\section{Photonic crystal structure}

\subsection{Photonic bandgap}

Photonic crystals have artificial structures consisting of materials with different permittivities. One of the essential features of photonic crystals is that when two materials with different permittivities are placed periodically under certain circumstances, a photonic bandgap 
can be obtained without allowing an electromagnetic wave with a specific frequency to exist. Thus, by defecting rows of the materials, the electromagnetic wave can propagate inside the defective area and not spread out. The photonic bandgap property makes it possible to create a waveguide that confines waves of specific frequencies. This property also enables us to design a frequency demultiplexer for two electromagnetic waves with two distinct frequencies.

Photonic crystals can be classified into three types: 1D, 2D, and 3D, depending on the number of directions in which the permittivity changes periodically. A 2D photonic crystal is periodic along two spatial axes, the $x$-axis and the $y$-axis, and uniform along a third spatial axis, the $z$-axis. It has bandgaps in the $x-y$ plane for specific values of the photonic structure. Using this bandgap property, we can design a photonic structure to confine input waves inside the defective area as no wave can exist outside. 2D photonic crystals can be simulated by creating a 2D periodic structure in the $x-y$ plane with an infinitely long lattice in the $z$-axis. Most of the unique properties and applications of photonic crystals can be discussed in a 2D structure. Therefore, in this study, we utilize 2D photonic crystals to discuss their properties in designing waveguides and demultiplexers.

A typical example of a 2D photonic crystal structure is demonstrated in Fig. 1. This example is based on a hexagonal lattice structure. Since square lattice provides different lattice space $R$ in setting photonic crystals, in this study, we adopt hexagonal lattice structures to keep equivalent $R$. Figure 1 indicates that the photonic crystal structure has two different permittivities, and they change periodically. The permittivity of vacuum is $\varepsilon_{0}=8.85418783 \times 10^{-12}$. Then, for example, the permittivity of photonic crystals can be set to $\varepsilon_{1}=11.9 \times \varepsilon_{0}$, periodically changing the permittivity in the photonic crystal structure. Here, $r$ represents lattice radius, and $R$ represents lattice spacing of each crystal. The bandgap of the input wave with a specific frequency can be obtained with appropriate $r$ and $R$. As a result, waves can be allowed to exist in the defective area while not being allowed to exist out of this area, creating a photonic crystal waveguide.

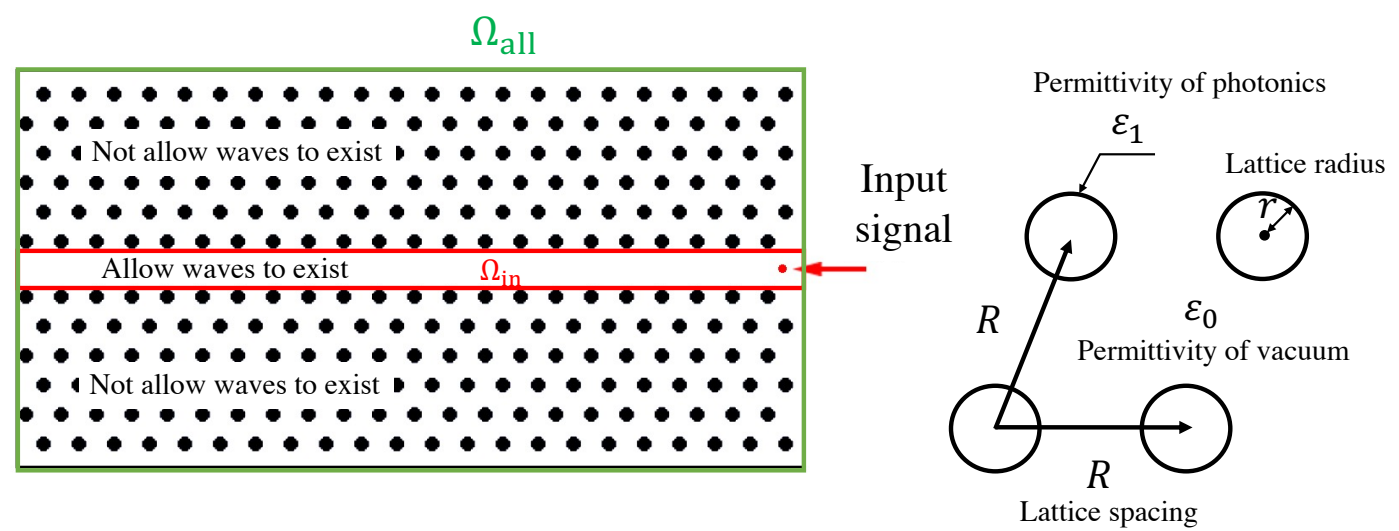

Figure 1: Photonic crystals using hexagonal lattice structure. 

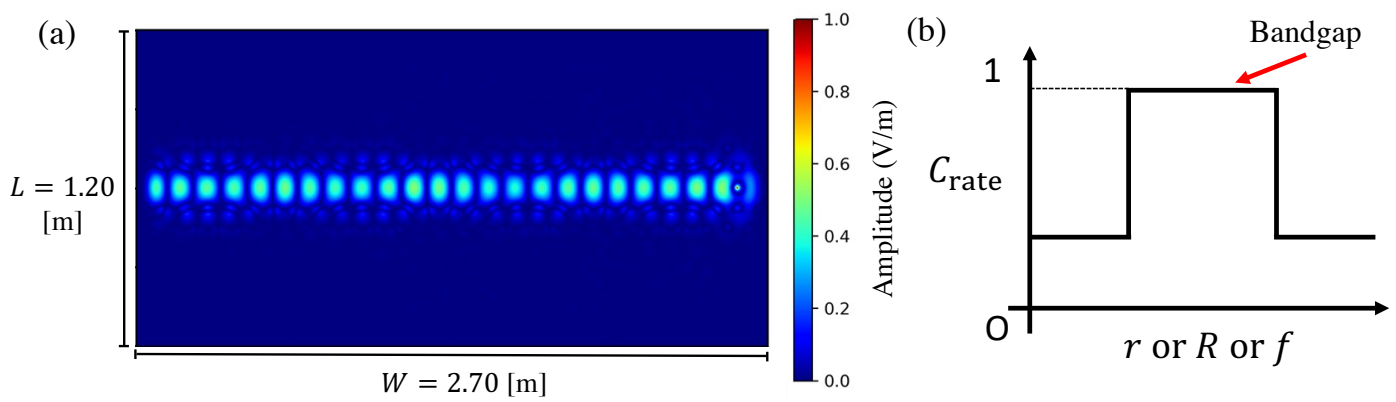

Figure 2: A photonic crystal waveguide using the bandgap property. (a) Distributions of the electric field simulated by FDTD. (b) $C_{\text {rate }}$ calculated based on Fig. 1 .

\subsection{Photonic crystal waveguide}

Based on the bandgap property of photonic crystals described above, applications in various fields are being considered. An electromagnetic wave with a frequency corresponding to the bandgap cannot exist inside the photonic crystal structure. However, suppose the periodic structure of the photonic crystal is partially broken to create defects. In that case, the wave is concentrated inside the defects. By using this property, it is possible to design optical waveguides using photonic crystals by defecting areas.

Figure 2 shows an example of a 2D photonic crystal waveguide using the defected structure shown in Fig. 1. This figure indicates that by intentionally adopting defects into the periodic structure of the photonic crystal in a straight line, the wave propagates in the defective area.

To investigate the bandgap property in the electromagnetic field, we employ the pointing vector $\boldsymbol{P}$ in this study. The pointing vector is the vector product of an electric field and a magnetic field. It is a physical quantity representing the density of the energy flow in an electromagnetic field. For electromagnetic waves, the direction of the pointing vector refers to wave direction of movement, and the intensity of the pointing vector refers to the energy density that passes through a unit area per unit time. Then, to find the bandgap of photonic crystal structures, we define a confinement rate $C_{\text {rate }}$ of the waveguide using the pointing vector. An example of the analysis model of the bandgap is shown in Fig. 1. When the area surrounded by the green frame is $\Omega_{\text {all }}$ and the area surrounded by the red frame of the defective part is $\Omega_{\text {in }}$, the wave confined in the waveguide can be measured by the confinement rate $C_{\text {rate }}$, as shown in (1).

$$
\begin{aligned}
& p_{\text {all }}=\int_{\Omega_{\text {all }}}\|\boldsymbol{P}\| d \Omega_{\text {all }} \\
& p_{\text {in }}=\int_{\Omega_{\text {in }}}\|\boldsymbol{P}\| d \Omega_{\text {in }} \\
& C_{\text {rate }}=\frac{p_{\text {in }}}{p_{\text {all }}}
\end{aligned}
$$

Thus, the structure of photonic crystal waveguide strongly depends on the combination 
of values of $R$ : lattice spacing, $r$ : lattice radius, and $f$ : frequency, as Fig. 2 demonstrated. By changing the three parameters to calculate each $C_{\text {rate }}$, appropriate structures can be found to design a frequency demultiplexer.

\section{Empirical mode decomposition}

\subsection{Intrinsic mode functions and residual}

To verify the performance of a designed demultiplexer, we use the empirical mode decomposition (EMD) to confirm if input waves are separated correctly or not in the instantaneous frequency domain. EMD decomposes a real-world signal $s(t)$ into a number of intrinsic mode functions (IMFs) and a residual called "trend," which can be defined as (2).

$$
s(t)=\sum_{i=1}^{n} c_{i}(t)+r(t)
$$

where $\sum_{i=1}^{n} c_{i}(t)$ is the IMFs set and $r(t)$ is the residual. Intrinsic mode functions are empirically defined as follows [15]:

- The number of extreme values of the signal and the number of zero intersections is equal, or the difference is 1 .

- At any given time, the average value of the upper envelope and the lower envelope is zero.

Moreover, the definition of the trend is empirically defined as follows [16]:

- A trend is a monotonic function that fits the data properties or a function with at most one extremum within a particular interval.

- Detrend is an operation to remove the trend, and volatility is data that removes the trend within a specific interval.

\subsection{Multivariate empirical mode decomposition}

EMD decomposes univariate signals using cubic spline without any mathematical proof. For details of EMD algorithm, please see [15]. After EMD has been proposed, many pieces of research expanded EMD from one to multiple variables to achieve comprehensive utilization. The multivariate EMD (MEMD) decomposes multivariate signals into their corresponding IMFs $[14,15]$.

For nonlinear and multi-channel signals such as photonic crystal prorogation simulated by FDTD method, MEMD can be considered a powerful tool for numerical analysis to decompose simulation results in the instantaneous frequency domain. In this research, we consider each cell of simulation results as multivariate signals to decompose and visualize photonic crystal prorogation using MEMD. Then, the MEMD algorithm for decomposing FDTD simulation results is demonstrated in Algorithm 1. 


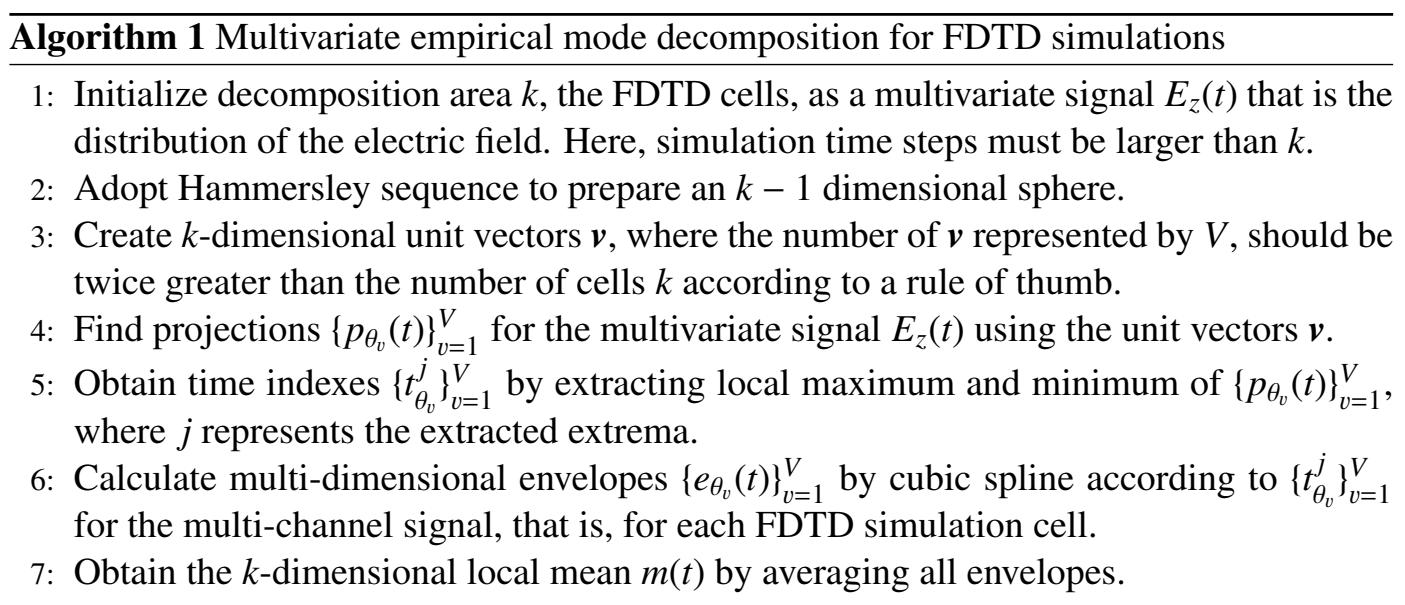

$$
m(t)=\frac{1}{V} \sum_{v=1}^{V} e_{\theta_{v}}(t)
$$

8: Extract nonlinear frequency components $c(t)$ using $c(t)=E_{z}(t)-m(t)$.

9: Initialize $c(t)$ as the multivariate signal and repeat steps 4-8 until the convergence condition [18] is satisfied. Then, add $c(t)$ as pseudo monochromatic waves to the IMF set.

10: Obtain residual $r(t)$ by $r(t)=E_{z}(t)-\sum_{i=1}^{n} c_{i}(t)$, where $c_{i}(t)$ is extracted IMFs. Initialize $r(t)$ as the multivariate signal and repeat 4-9 unless $r(t)$ satisfy the definition of trend.

\subsection{Hilbert spectrum analysis}

After MEMD decomposes a simulation result, the Hilbert transform (HT) [17] is applied to each IMF to calculate the instantaneous amplitude and frequency of electromagnetic waves. The Hilbert spectrum analysis (HSA) schemes a figure with time on the horizontal axis, frequency on the vertical axis, and amplitude in color [15].

By plotting frequencies and amplitudes of all IMFs in time series, instantaneous frequencies and amplitudes of electromagnetic waves are revealed. Thus, after decomposing FDTD simulation results using MEMD, the propagation of electromagnetic waves can be analyzed in the instantaneous frequency domain using HSA.

\section{Results and evaluations}

\subsection{Photonic crystal frequency demultiplexer design}

In this section, the bandgap property is adopted to find three different structures to propagate and separate two different frequency waves. To find appropriate parameters for designing frequency demultiplexer made of photonic crystals, we simulate waveguides based on varied parameters (frequency, lattice radius, and lattice spacing) using 2D FDTD (TM mode).

Table 1 shows the parameters of each waveguide. Frequency is changing from $0.1[\mathrm{GHz}]$ to 2.0 [GHz]. In this study, to satisfy Courant-Friedrichs-Lewy Condition, we determine the color cell size of FDTD simulation $\Delta x=7.49 \times 10^{-3}[\mathrm{~m}]$ according to the wave that has 
Table 1: Detecting photonic bandgaps simulated by FDTD (TM mode)

\begin{tabular}{ll}
\hline Parameters & Values \\
\hline Frequency & $f=0.1-2.0[\mathrm{GHz}]$ \\
Lattice radius & $r=7.49 \times 10^{-3}-67.45 \times 10^{-3}[\mathrm{~m}]$ \\
Lattice spacing & $R=4 r-8 r[\mathrm{~m}]$ \\
Light speed & $c=2.299792458 \times 10^{8}[\mathrm{~m} / \mathrm{s}]$ \\
Time variation & $\Delta t=159.1[\mathrm{~ns}]$ \\
Wavelength & $\lambda=c / f[\mathrm{~Hz}]$ \\
Permittivity & $\varepsilon_{0}=8.85418783 \times 10^{-12}$ \\
& $\varepsilon_{1}=11.9 \times \varepsilon_{0}$ \\
Analysis area & $\Delta x=7.49 \times 10^{-3}[\mathrm{~m}]$ \\
& $W=2.70[\mathrm{~m}]$ \\
& $L=1.20[\mathrm{~m}]$ \\
Absorption boundary condition (PML) & Up down left right $0.15[\mathrm{~m}]$ \\
FDTD simulation cells & $W_{\text {cell }}=360+40(\mathrm{PML})$ \\
& $L_{\text {cell }}=160+40(\mathrm{PML})$ \\
\hline
\end{tabular}

the highest frequency $f=2.0[\mathrm{GHz}]$ by dividing its wavelength by 20 cells. Then time variation is $\Delta t=159.1$ [ns]. Consequently, lattice radius is changing from $7.49 \times 10^{-3}[\mathrm{~m}]$ to $67.45 \times 10^{-3}[\mathrm{~m}]$, and lattice spacing is changing from 4 to 8 times lattice radius. The analysis area is set to $W=2.70[\mathrm{~m}]$ and $L=1.20[\mathrm{~m}]$, as shown in Fig. 2, for simulating a waveguide using one of the parameter sets, lattice radius: $r$, lattice spacing: $R$, and input wave frequency: $f$. In addition, PML (perfectly matched layer) is set at the top, bottom, left and right of the boundary condition.

After simulating all parameters shown in Table 1, 20 bandgap maps of photonic crystal waveguides for each electromagnetic wave with specific frequencies (from 0.1 [GHz] to 2.0 [GHz]) are obtained. Figure 3 shows two selected bandgap maps of two different frequency waves, $f_{1}=1.5[\mathrm{GHz}]$ and $f_{2}=2.0[\mathrm{GHz}]$. The horizontal axis represents the lattice spacing $R$, and the vertical axis represents the lattice radius $r$, and the color represents the confinement rate $C_{\text {rate }}$ defined above. As shown in figures, different frequency waves have different distributions of $C_{\text {rate }}$. Furthermore, two figures also demonstrate that three different types of the bandgap were found by dividing $r$ into 9 cells, which is enough to design a demultiplexer. Therefore, we can adopt this feature to design two different structures to separate the two frequency waves.

To design and simulate a demultiplexer, three different structures employing corresponding bandgaps are necessary. One structure can propagate the composited wave with both two frequencies $f_{1}$ and $f_{2}$, while other structures only can propagate the wave with frequencies $f_{1}$ or $f_{2}$, respectively. As shown in Fig. 3, (A) red frame indicates that $f_{1}=1.5[\mathrm{GHz}]$ wave is confined with high $C_{\text {rate }}$ in the bandgap while $f_{2}=2.0[\mathrm{GHz}]$ wave is not, corresponding to $r=22.47 \times 10^{-3}[\mathrm{~m}]$ and $R=97.37 \times 10^{-3}[\mathrm{~m}]$. (B) Green frame indicates that $f_{1}=1.5$ [GHz] wave is confined with high $C_{\text {rate }}$ in the bandgap while $f_{2}=2.0[\mathrm{GHz}]$ wave is not, corresponding to $r=14.98 \times 10^{-3}[\mathrm{~m}]$ and $R=74.91 \times 10^{-3}[\mathrm{~m}]$. (C) Black frame indicates that both of $f_{1}=1.5[\mathrm{GHz}]$ and $f_{2}=2.0[\mathrm{GHz}]$ waves are confined with high $C_{\text {rate }}$ in the 


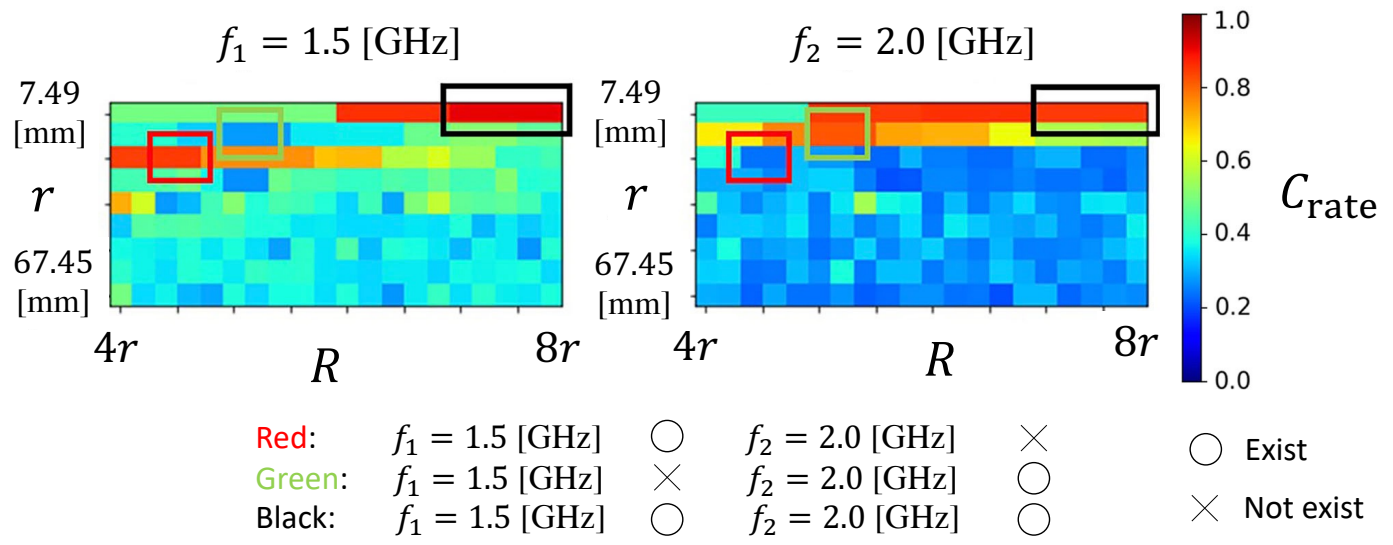

Figure 3: Two bandgap maps with $C_{\text {rate }}$ for two different frequencies in photonic crystal waveguides by changing the lattice radius $r$ and the lattice spacing $R$.

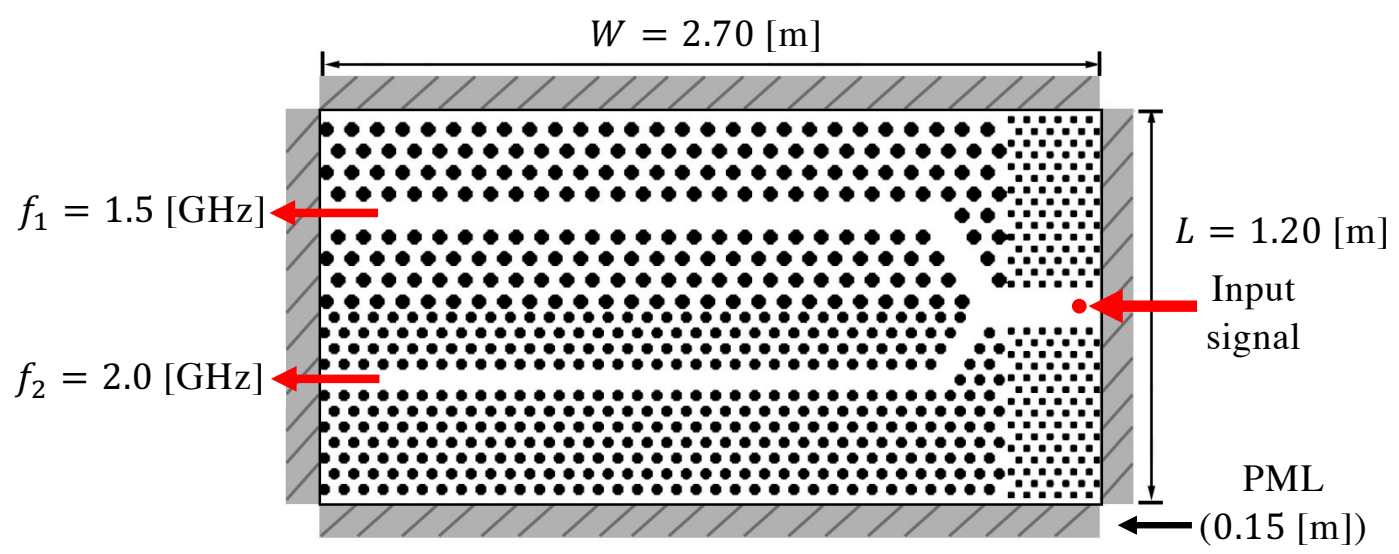

Figure 4: A designed photonic crystal demultiplexer based on Fig. 3.

bandgap, corresponding to $r=7.49 \times 10^{-3}[\mathrm{~m}]$ and $R=59.92 \times 10^{-3}[\mathrm{~m}]$. Hence, we adopt the three structures to design a demultiplexer indicated in Fig. 4. By defecting one row from each of up and down bandgap structures, two waveguides for separately propagating $f_{1}=1.5[\mathrm{GHz}]$ and $f_{2}=2.0[\mathrm{GHz}]$ are obtained.

In this frequency demultiplexer made of photonic crystals, the analysis area is set to $W=2.70[\mathrm{~m}]$ and $L=1.20[\mathrm{~m}]$, and PML (perfectly matched layer) is set at the top, bottom, left and right of the boundary condition with $0.15[\mathrm{~m}]$, respectively. The time variation is $\Delta t=159.1[\mathrm{~ns}]$, as same as the waveguide simulation introduced above. The signal source $E_{z}(t)$ is composited by two different frequency waves $f_{1}=1.5[\mathrm{GHz}]$ and $f_{2}=2.0[\mathrm{GHz}]$, as shown in (3).

$$
E_{z}(t)=\sin \left(\pi f_{1} t\right)+\sin \left(\pi f_{2} t\right)
$$

Figure 5 shows that the composited signal (3) is inputted from the left of the demultiplexer and separated into two different waves on the right, where time $t=954.6[\mu \mathrm{s}]$ is 


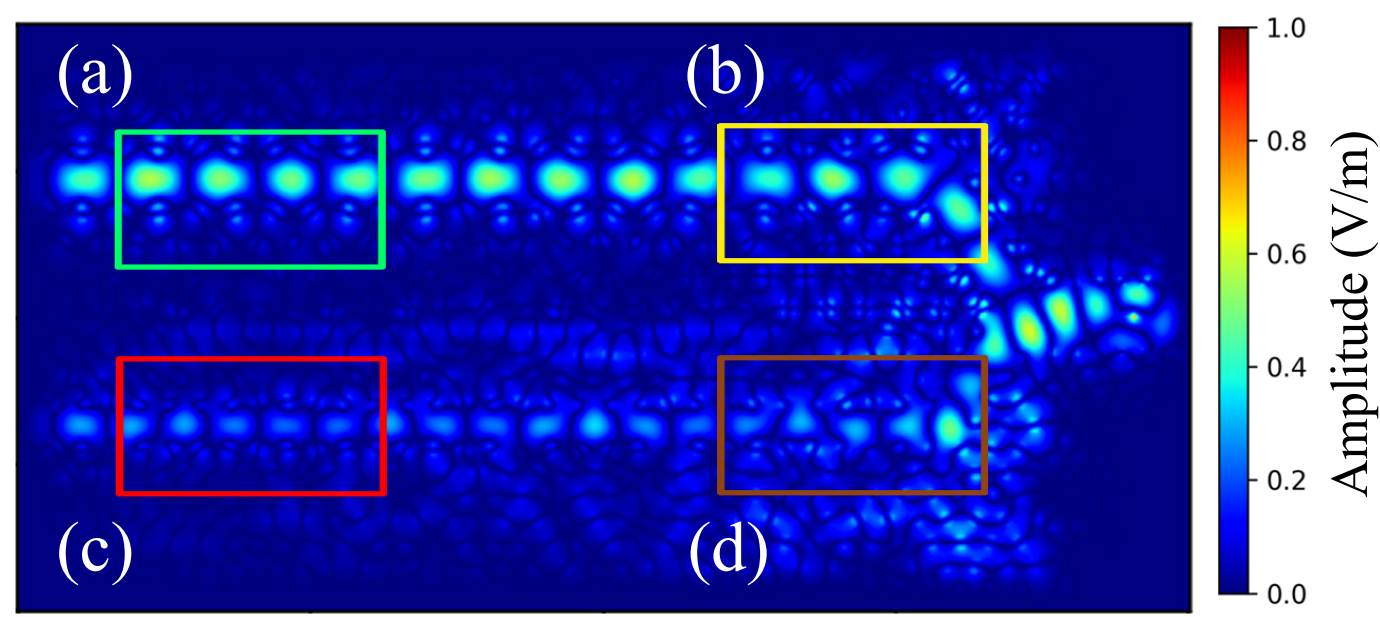

Figure 5: Electromagnetic wave propagation of the frequency demultiplexer simulated by FDTD in TM mode $E_{z}(t=954.6[\mu \mathrm{s}])$.

selected after achieving a stationary state. The composited wave is propagating in the entrance and separating due to the different structures. The upper is corresponding to $f_{1}=1.5$ [GHz], and the lower is corresponding to $f_{2}=2.0[\mathrm{GHz}]$, respectively. We can see from Fig. 5 that there is no leaking in the entrance while the wave is spearing outside after the entrance. This indicates that two waves are propagating collectively in the entrance, and $f_{1}=1.5[\mathrm{GHz}]$ wave is leaking in the lower range, and $f_{2}=2.0[\mathrm{GHz}]$ wave is leaking in the upper range.

\subsection{Evaluating the frequency demultiplexer simulation results}

In Fig. 5, electric field regions near the entrance are indicated by yellow frame (b) and brown frame (d), and the electric field regions near the exit are indicated by green frame (a) and red frame (c). To demonstrate that the pointing vector can be adopted to evaluate bandgaps for specific frequency waves, we plot pointing vectors of the designed frequency demultiplexer results shown in Fig. 6. Black circles represent photonic crystals, and the arrows represent pointing vectors $\boldsymbol{P}$, the size and color of the arrows indicate the energy $\|\boldsymbol{P}\|$. As shown in Fig. 6 (a) and (c), almost all pointing vectors are confined in the defected region of the designed frequency demultiplexer by their photonic crystal structures, respectively. Therefore, the lattice radius $r=22.47 \times 10^{-3}[\mathrm{~m}]$ and the lattice spacing $R=97.37 \times$ $10^{-3}[\mathrm{~m}]$ of this photonic crystal structure correctly propagates an electromagnetic wave with frequency $1.5[\mathrm{GHz}]$. Meanwhile, the lattice radius $r=14.98 \times 10^{-3}[\mathrm{~m}]$ and the lattice spacing $R=74.91 \times 10^{-3}[\mathrm{~m}]$ of this photonic crystal structure correctly propagates an electromagnetic wave with frequency $2.0[\mathrm{GHz}]$. Thus, the propagation based on the photonic crystal bandgap property can be analyzed and evaluated in the time domain by visualizing the pointing vector in the electromagnetic field.

To evaluate the performance of the demultiplexer in the frequency domain, MEMD is adopted to verify and visualize electromagnetic waves in each region by treating the sim- 


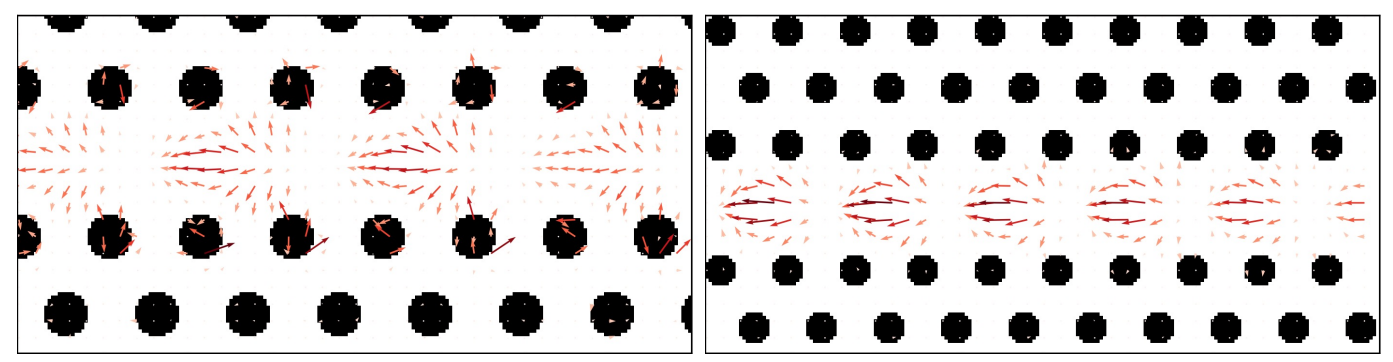

Figure 6: Pointing vector maps of the designed frequency demultiplexer simulation in Fig. 5. (a) Green frame, (c) Red frame. (Two figures are recalculated for achieving higher resolution.)

ulation results of electric fields as multi-channel signals in Fig. 5 (a-d). All regions are considered as multivariate signals and decomposed by MEMD, respectively. The FDTD cells of each region are 5,000 channels and the time steps are from 5,000 to 11,000 after the simulation becomes stable.

After MEMD decomposes the result, instantaneous frequencies and amplitudes of photonic crystal prorogation in each region are obtained using HT. Figure 7 is the Hilbert spectrum of each region. Due to the property of MEMD that all channels are decomposed into the same number of IMFs, we only show one channel in the center of the region to discuss the results. Figure 7 (a) represents the Hilbert spectrum obtained from the green frame in Fig. 5 (a), which is near the exit on the left. Figure 7 (b) represents the Hilbert spectrum obtained from the yellow frame in Fig. 5 (b), which is near the entrance on the right. As the up region is corresponding to propagate $f_{1}=1.5[\mathrm{GHz}]$, the $f_{1}=1.5[\mathrm{GHz}]$ wave is clearly shown in the both spectra. The region near the entrance has much more IMFs (nonlinear modes) than the region near the exit by comparing both spectra. This reveals that the $f_{2}=2.0[\mathrm{GHz}]$ has been removed after the entrance.

Figure 7 (c) represents the Hilbert spectrum obtained from the red frame in Fig. 5 (c), which is near the exit. Figure 7 (d) represents the Hilbert spectrum obtained from the brown frame in Fig. 5 (d), which is near the entrance. As the low region is corresponding to propagate $f_{2}=2.0[\mathrm{GHz}]$, the $f_{2}=2.0[\mathrm{GHz}]$ wave is clearly shown in the both spectra. The result is similar to the up region, which is that the region near the entrance has much more IMFs (nonlinear modes) than the region near the exit.

Since we inputted the same channels (FDTD cells) to decompose simulation results using MEMD, and the waveguide area in Fig. 5 (c) is smaller than the waveguide area in Fig. 5 (a), a nonlinear wave near $1.0[\mathrm{GHz}]$ was still decomposed by MEMD, as shown in 7 (c). However, the amplitude of this nonlinear wave is relatively smaller than the amplitude of 2.0 [GHz]. Thus, by comparing Fig. 7 (a) and Fig. 7 (b), Fig. 7 (c) and Fig. 7 (d), the demultiplexer works correctly and the composited wave is demultiplexed into $f_{1}$ and $f_{2}$, respectively. 

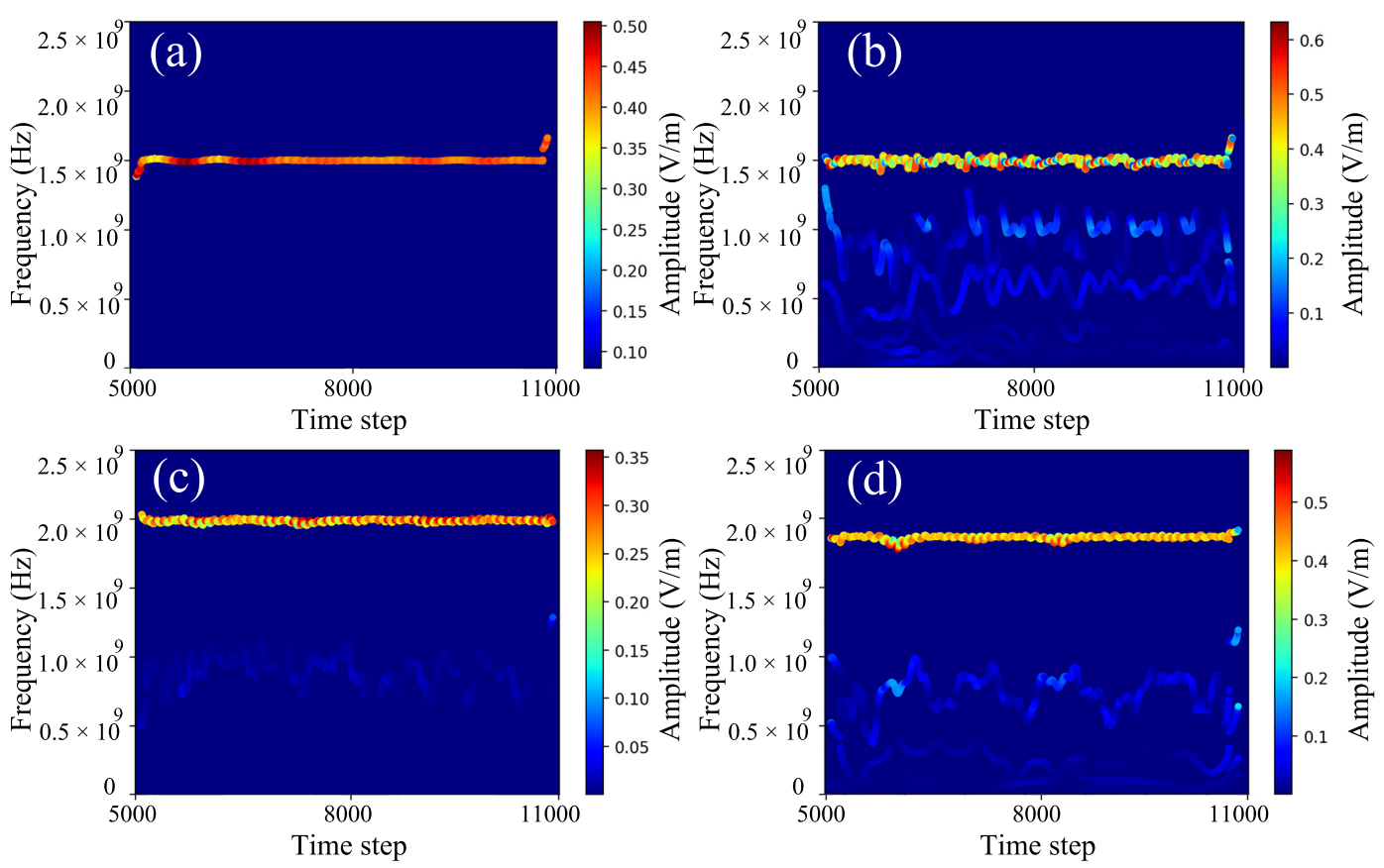

Figure 7: Hilbert spectra of decomposed IMFs by MEMD for each region in Fig. 5. (a) green frame, (b) yellow frame, (c) red frame, (d) brown frame.

\section{Conclusions}

Photonic crystals are artificially created, and their permittivity is periodically changed. If the period is appropriately designed based on the bandgap property, it becomes possible to propagate the distinct frequency electromagnetic wave. Hence, photonic crystals can be employed to develop optical devices such as waveguides and demultiplexers. In this paper, photonic bandgaps are adopted to design a frequency demultiplexer made of photonic crystals simulated by FDTD method. Additionally, this study discusses the applicability of MEMD to the simulation results of the frequency demultiplexer. The conclusions of this study can be summarized as follows:

- Frequency demultiplexers can be designed using photonic crystals to separate waves with their specific frequencies based on bandgap properties.

- Photonic crystal bandgaps for designing frequency demultiplexers can be obtained and visualized using pointing vectors of the electromagnetic field simulated by FDTD.

- The simulation results generated by FDTD can be decomposed and visualized by treating cells of the electric field as multivariate signals into MEMD.

- The HSA can be used to evaluate the performance of photonic crystal demultiplexers in the instantaneous frequency domain.

Although the EMD has no mathematical proof, the results of this study showed the possibility of decomposing and analyzing electromagnetic wave propagation using MEMD. 
Journal of Advanced Simulation in Science and Engineering

Then, the accuracy of MEMD in the analysis of electromagnetic simulations by FDTD and its further applications are considered to be future issues.

\section{References}

[1] Yasumoto, K., Electromagnetic theory and applications for photonic crystals, CRC press, 2018.

[2] Rostami, A., Nazari, F., Banaei, H. A., and Bahrami, A, A novel proposal for DWDM demultiplexer design using modified-T photonic crystal structure. Photonics and Nanostructures-Fundamentals and Applications, 8(1), 14-22, 2010.

[3] Sullivan, D. M, Electromagnetic simulation using the FDTD method, John Wiley \& Sons, 2013.

[4] Kawaguchi, H., and Kodate, S., Method of real time simulation for design of microwave devices based on FDTD method, Journal of Advanced Simulation in Science and Engineering, 2(2), 225-234, 2015

[5] Itoh, T., and Ikuno, S., Efficient simulation of electromagnetic wave propagation in complex shaped domain by hybrid method of FDTD and MTDM based on interpolating moving least squares method. IEEE Transactions on Magnetics, 53(6), 1-4, 2017.

[6] T. Hanawa and S. Ikuno, Large-Scale Simulation for Optical Propagation in 3D Photonic Crystal Using the FDTD Method With Parallel Processing, in IEEE Transactions on Magnetics, vol. 43, no. 4, pp. 1545-1548, 2007.

[7] Fujita, Y., Ikuno, S., Kubo, S., and Nakamura, H., Finite-difference time-domain analysis of electromagnetic wave propagation in corrugated waveguide: Effect of miter bend/polarizer miter bend, Japanese Journal of Applied Physics, 55(1S), 01AH06, 2015.

[8] Sheen, D. M., Ali, S. M., Abouzahra, M. D., and Kong, J. A., Application of the three-dimensional finite-difference time-domain method to the analysis of planar microstrip circuits, IEEE Transactions on microwave theory and techniques, 38(7), 849857,1990

[9] Hsu, T. T., and Carin, L., FDTD analysis of plane-wave diffraction from microwave devices on an infinite dielectric slab, IEEE microwave and guided wave letters, 6(1), 16-18, 1996.

[10] Feng, D., Wang, X., and Zhang, B., Specific evaluation of tunnel lining multi-defects by all-refined GPR simulation method using hybrid algorithm of FETD and FDTD, Construction and Building Materials, 185, 220-229, 2018.

[11] Huang, N.E., Shen, Z., Long, S.R., Wu, M.C., Shih, H.H., Zheng, Q., Yen, N.C., Tung, C.C., and Liu, H.H., The empirical mode decomposition and the Hilbert 
spectrum for nonlinear and non-stationary time series analysis, Proceedings of the Royal Society of London. Series A: Mathematical, Physical and Engineering Sciences, 454(1971), pp. 903-995, 1998.

[12] Rilling, G., Flandrin, P., Gonçalves, P., and Lilly, J.M., Bivariate empirical mode decomposition, IEEE signal processing letters, 14(12), pp. 936-939, 2007.

[13] ur Rehman, N., and Mandic, D.P., Empirical mode decomposition for trivariate signals, IEEE Transactions on signal processing, 58(3), pp. 1059-1068, 2009.

[14] Rehman, N., and Mandic, D.P., Multivariate empirical mode decomposition, Proceedings of the Royal Society A: Mathematical, Physical and Engineering Sciences, 466(2117), pp. 1291-1302, 2009.

[15] Huang, N.E,. and Shen, Z., Hilbert-Huang transform and its applications, World Scientific, 2014.

[16] Wu, Z., Huang, N. E., Long, S. R., and Peng, C. K., On the trend, detrending, and variability of nonlinear and nonstationary time series. Proceedings of the National Academy of Sciences, 104(38), 14889-14894, 2007.

[17] Bracewell, R. N. and Bracewell, R. N., The Fourier transform and its applications, McGraw-Hill New York, 1986.

[18] Huang, N. E., Wu, M. L. C., Long, S. R., Shen, S. S., Qu, W., Gloersen, P., and Fan, K. L., A confidence limit for the empirical mode decomposition and Hilbert spectral analysis, Proceedings of the Royal Society of London. Series A: Mathematical, Physical and Engineering Sciences, 459(2037), 2317-2345, 2003. 